\title{
3D Heterogeneous Integration Strategy for Physically Flexible CMOS Electronic Systems
}

\author{
Sohail Faizan Shaikh \\ mmh Labs, Electrical Engineering \\ King Abdullah University of Science \\ and Technology (KAUST) \\ Thuwal, Saudi Arabia \\ SohailFaizan.Shaikh@kaust.edu.sa \\ ORCID ID: 0000-0001-7640-0105
}

\author{
Nazek El-Atab \\ mmh Labs, Electrical Engineering \\ King Abdullah University of Science \\ and Technology (KAUST) \\ Thuwal, Saudi Arabia \\ Nazek.Elatab@kaust.edu.sa \\ ORCID ID: 0000-0002-2296-2003
}

\author{
Muhammad Mustafa Hussain \\ mmh Labs, Electrical Engineering, \\ King Abdullah University of Science \\ and Technology (KAUST), Thuwal, \\ Saudi Arabia, \\ EECS, University of California, \\ Berkeley, CA, USA \\ MuhammadMusatafa.Hussain@kaust.e \\ du.sa mmhussain@ berkeley.edu \\ ORCID ID: 0000-0003-3279-0441
}

\begin{abstract}
Flexible and stretchable electronics has emerged as a promising field in the past decade, especially towards healthcare and environmental monitoring applications. We have witnessed significant progress in developing flexible sensors, logic/memory devices (transistors), communication modules, energy sources, and alternate materials to enhance performance with added physical conformity. However, there are unaddressed challenges in matching status-quo CMOSbased electronics, packaging, reliable flexible interconnects, and lack of pragmatic integration schemes to readily complement the existing state-of-the-art technology. Moreover, often rigid ICs are used on flexible polymeric substrates placed sparsely, making the system bendable with a caveat of reduced area efficiency. Hence, we present a CMOS compatible heterogeneous integration scheme with a unique 3D coin architecture to obtain truly flexible electronic systems. This integration sequence has multiple polymeric layers that resemble a coin with two faces and a sandwich layer. The bottom face of the coin hosts flexible sensors while the top face is reserved for communication (antenna) and energy harvester (flexible solar cell), and the associated electronic ICs (microcontroller, RF IC, solid-state micro-batteries) are embedded in the sandwich layer (all components in the flexible form). We use the modular-LEGO approach for the assembly of bare die ICs like a lock-and-key mechanics in plug and play manner to obtain reliable, flexible interconnects and accurate assembly. These multiple layers are connected electrically using vertical through-polymer-via (TPV) that shows less than $4 \%$ overall change in the resistance across the longest metallic interconnect layer trace. This vertical TPV interconnection helps in maximizing area usage, thereby increasing area efficiency. We transformed the commercial CMOS based 16 MHz microprocessor bare die and an array of micro-batteries with a rating of $5 \mu \mathrm{Ah} @ 3.9 \mathrm{~V}$ into ultrathin $(<20 \mu \mathrm{m})$ flexible form after assembly of thicker dies $(200-300 \mu \mathrm{m})$ into the LEGO sites. It can integrate a solar cell on top face after following the corrugation approach of converting rigid solar cells into a flexible one. At the same time, the antenna for communication and the TPV are fabricated using electrochemical deposition technique. The overall thickness of the 3D-coin system is less than $1 \mathrm{~mm}$ and free from any rigid component making it flexible up to a bending radius of $\sim 1 \mathrm{~mm}$. Soft-polymeric encapsulation provides mechanical strength, reduces stresses, and improves ruggedness for the stand-alone electronic system. Thus, we believe our 3D heterogeneous integration strategy offers a pragmatic approach to obtaining truly flexible electronic systems without compromising next-generation electronic systems' performance aspects.
\end{abstract}

Keywords-3-D integration, flexible electronics, internet-ofthings, CMOS, soft-encapsulation

\section{INTRODUCTION}

We are living in the age of Internet of Things (IoT) and Internet of Everything (IoE) that promises seamless connection of device-data-people-process. An efficient IoT/IoE system needs a pragmatic approach to integrate billions of sensors on complex, irregular and challenging biological and non-living surfaces. Flexible sensors, transistors, and other individual devices have been shown in past [1]-[4], in addition to hybrid systems where rigid ICs are populated on flexible polymeric substrate [5]-[7]. In parallel, huge efforts going on in the emerging field of flexible electronics, exploring 0D/1D/2D materials, organic materials, polymers, and low-cost processing techniques. Nonetheless, for data management conventional rigid ICs are used [7]-[9]. Additionally, different approaches have been explored to use self-assembled layers, thinned devices, micro-bumps, specific bonding layer and high-temperature process-based pressure application for stack bonding. A variety of methods are used to reduce the CMOS devices into a thin and flexible form [1], [10]-[16]. Furthermore, the scaling continues to impose unprecedented challenges and hence active utilization of $\mathrm{z}$ axis has seen tremendous growth in recent past. Stacking ICs without substantial thickness reduction for through-silicon-via (TSV) restricts efficient area utilization and yields thicker stack thereby losing flexibility [17]-[22]. In-plane single layer assembly of active and passive electronics causes severe skin/cell inflammation by heat dissipation from active devices. Therefore, robust integration strategy is required for a reliable physically compliant electronic system. One of the major challenges in integration is having reliable flexible interconnects that do not delaminate or fracture at the interface of rigid ICs and flexible substrate. Therefore, robust integration strategy is required for obtaining a reliable physically compliant electronic system overcoming these challenges.

To overcome these challenges, here we present a 3D coin architecture based heterogeneous integration scheme to obtain a flexible standalone CMOS electronic system having array of CMOS ICs, sensors, battery, and RF antenna (Fig. 1). In a 3Dcoin architecture, heterogeneous materials and technologies like CMOS, polymers, and sensors are integrated together on a single platform [15]. The 3D coin architecture can host multiple sensors (for example temperature, pressure, humidity etc.) on one side of the coin interacting with processing 
electronics sandwiched in the polymeric platform using through-polymer-via (TPV). A sequential-etch-back (SEB) technique is used to transform state-of-the-art CMOS ICs into flexible ICs, converted into flexible LEGO for secure identification and accurate assembly using modular Legoelectronics approach like a plug-and-play (or lock-and-key) mechanism [15]. The top face of the coin architecture is reserved for antenna for data communication (Fig. 2d) everything in the embedded soft packaging. The polymeric encapsulation for the electronic interface ensures the biocompatibility as well as ruggedness [23], [24]. In addition, this coin-architecture provide physical isolation of the electronics and communication element from the sensory layer thereby not interacting with the host body for various monitoring applications.

\section{Heterogenous 3D COIN ARChITECTURE INTEGRATION}

Complete fabrication schematic for 3D heterogeneous coin integration is illustrated in Fig. 1. We start with spinning a thin $(<10 \mu \mathrm{m})$ polyimide (PI) gradually curing in steps $(90-$ $300{ }^{\circ} \mathrm{C}$ ) on a bulk silicon (100) carrier wafer. This provides mechanical strength to the flexible system and acts as a host substrate for Lego-based lock-and-key assembly. Next, a thin sputtered metal $(\mathrm{Cu}, 100 \mathrm{~nm})$ forms circuit interconnects on PI followed by spinning second layer of $10 \mu \mathrm{m}$ thin PI as binding layer and TPV etched to form female Lego site for multiple die assembly. Etching is performed in multiple steps of lithography, patterning, and protecting the critical areas using a hard mask of Al metal. This patterning of PI is previously reported from different works on flexible sensors on PI [24]-[28]. A $280 \mu \mathrm{m}$ thick microcontroller MSP430 ${ }^{\mathrm{TM}}$ (8kB Flash and 256B RAM) from Texas Instruments and solid-state micro-batteries $\left(\right.$ Cymbet $^{\mathrm{TM}} \mathrm{CBC} 005$; thickness $=$ $200 \mu \mathrm{m}$ ) are assembled (Fig 2A) in the Lego sites using a modular-LEGO electronics approach[15] that uses softvacuum suction-based pick-and-place tool for high yield (7000 UPH) with a placement accuracy of $\pm 7 \mu \mathrm{m}$. The conversion of rigid IC to flexible $(<20 \mu \mathrm{m}$ thickness) is achieved by sequential etch back using DRIE while the PDMS cured act as sidewall spacer protecting the lateral etching. TPV for the antenna and energy harvesting (solar cell) are formed by using $\mathrm{CO}_{2}$ laser ablation on encapsulated PDMS layer and filled with ECD $\mathrm{Cu}$ for vertical connection.

The flexed ICs are encapsulated with $100 \mu \mathrm{m}$ PDMS and we flip it before transferring it on a carrier wafer having cured PDMS. TPVs are etched through PI and PDMS interface to make vertical interconnections from the microprocessor, battery, and antenna. PI and PDMS are etched at $60{ }^{\circ} \mathrm{C}$ and $10^{\circ} \mathrm{C}$ respectively, with varying gaseous mixture of $\mathrm{CF}_{4}: \mathrm{O}_{2}$ in the ratio of $1: 10$ and 1:5 respectively in RIE. TPVs $(14 \mu \mathrm{m}$ deep) are filled with $\mathrm{Cu}$ using electrochemical-deposition (ECD). $15 \mu \mathrm{m} \mathrm{ECD} \mathrm{Cu}$ is grown in TPVs (Fig. 3), which lands on the pads of MCU and battery dies. Finally, the systems is encapsulated with another $100 \mu \mathrm{m}$ PDMS to neutralize possible mechanical stress mismatch from the top and bottom side of the 3D-coin sandwich structure. Thus a fully complaint 3D electronic system is obtained which can be seen flexed around $3 \mathrm{~mm}$ bending radius folded in the finger in Fig. 2 .

\section{RESULTS AND DISCUSSIONS}

To validate the integration sequence, we have performed the electrical characterization of MCU and sensors in varying conditions to understand the process-induced variations and studied the effect of volumetric thickness reduction.

\section{A. Electrical Characterisation of LEGO Interconnects}

Embedded structure, TPV coverage and protruding pads at different faces are clearly visible under SEM (Fig 3) signifying the seamless continuity of vertical interconnects. For electrical characterization of interconnects, IV curve and change in resistance is measured for LEGO piece, at multiple TPV, and after assembly in the line (Fig 4 a). It is evident from the plots that the resistance observed at multiple TPV test structures and when assembled in the Lego form has insignificant variation since, the overall maximum change in resistance observed after assembly was $<0.15 \Omega$ across via. This establishes that the Lego interconnection works for the desired applications without any performance degradation. Next step is to check process reliability for flexing MCU ICs.

\section{B. Electrical Characterisation of the Microcontroller}

The current consumption of the reference microcontroller unit and processed dies is measured. Variation in current consumption of bare die thick $(280 \mu \mathrm{m})$ IC is recorded during code writing, in the idle state after writing, and during the code run sequence. The same measurements are recorded for the MCU after reducing the thickness to less than $15 \mu \mathrm{m}$ and after making TPVs for vertical interconnects. From Fig. 4b, the average current consumption of LED programmed to blink is $3.3 \mathrm{~mA}$ and that of MCU in active state is $\sim 0.400 \mathrm{~mA}$ for an unprocessed die. The active state average current consumption of thinned die and the die after TPV processing did not change and the values read $\sim 0.402 \mathrm{~mA}$ and $\sim 0.405 \mathrm{~mA}$, respectively. The currents in the idle state after coding the sequence drop to values of $0.108 \mathrm{~mA}, 0.111 \mathrm{~mA}$ and $0.111 \mathrm{~mA}$, respectively.

Similarly, the same average current consumptions of the MCU and integrated LED has been measured to understand mechanically induced variations due to bending. And we have observed that the current consumption of thin MCU changes negligibly from $\sim 0.402 \mathrm{~mA}$ to $\sim 0.405 \mathrm{~mA}$ in the bent state (bending radius $5 \mathrm{~mm}$ ) in active mode. The same values drop to $\sim 0.111 \mathrm{~mA}$ to $0.109 \mathrm{~mA}$ in the idle state after writing the code sequence. Thus, we infer that our integration sequence is the most pragmatic route for the flexible electronic based IoT and IoE applications.

\section{Sensor Characterisation}

Implementation of IoT and IoE rely on the integration of the different standalone sensory systems. Hence, we have fabricated sensing modality that are often required for healthcare applications like temperature, and humidity sensors. The process is replicated from previously published flexible temperature sensors. The performance of fabricated temperature sensors on front (top) face of coin is compared with a commercially available reference sensor from Sensiron $^{\mathrm{TM}}$. We can correlate from Fig. $5 \mathrm{~b}$ that the response of both the test and the reference sensors follow same trend. The measurements for the variations in the fabricated sensor on the front face (top) of the 3D-coin interface are very much identical to the commercial sensors, however, the focus of the work is not on the sensor performance itself. For multiple sensors integration, an efficient multiplexing technique can be used to acquire the data from hundreds of sensory electrodes. Individuals or groups of electrodes can be accessed using multi-level multiplexer architecture with connected using TPV. An antenna can be designed to match a suitable 
frequency as that of desired communication protocol ranging from near-field communication, bluetooth, or Zig-Bee. And this can be easily fabricated with this integration strategy. We have also demonstrated a simple application where the standalone system is mounted on the finger which has 4 batteries, MCU, LED, and temperature sensor (Fig. 5a). This wearable stand-alone system detects the temperature threshold and warns the user whenever there is a increase in temperature above $25{ }^{\circ} \mathrm{C}$. This experiment for increasing has been performed using a yellow desk lamp to bring in close vicinity for increasing the temperature while room temperature was set to $20^{\circ} \mathrm{C}$.

\section{CONCLUSION}

Heterogeneous integration of materials, processes, and technologies is the way to go forward for the efficient implementation of IoT and IoE applications. Therefore, we have presented a low temperature, CMOS compatible heterogeneous integration scheme, which can give a fully compliant electronic system free from any rigid component and in 3D coin architecture for maximized area efficiency and reduced thermal impact on skin tissues/cells. We have reported the reliable process of making IC flexible using SEB (final thickness $<20 \mu \mathrm{m}$, and bending radii of less than $1 \mathrm{~mm}$ ), without any performance degradation. Integration of graphene-based $24 \mathrm{GHz}$ antenna, sensors array, and processing unit is reported. This is the most pragmatic and feasible approach to the implementation of futuristic applications augmenting life.

Heterogeneous integration of materials, processes, and technologies is the way to go forward for the efficient implementation of IoT and IoE applications. Therefore, we have presented a low temperature, CMOS compatible heterogeneous 3D integration scheme, which can give a fully compliant electronic system free from any rigid component. This novel 3D-coin architecture enables maximized area efficiency and reduced thermal impact on skin tissues/cells. We have reported that commercial IC can be turned into flexible LEGO using SEB (final thickness $<20 \mu \mathrm{m}$, and bending radii of less than $1 \mathrm{~mm}$ ), without any performance degradation. Overall reliability of integration scheme and interconnect has been demonstrated by system demonstration. We believe, our 3D coin architecture based integration scheme is the most pragmatic and feasible approach to the implementation of futuristic applications augmenting life.

TABLE I. TABLE TYPE STYLES

\begin{tabular}{|c|c|c|c|}
\hline \multirow{2}{*}{$\begin{array}{c}\text { Compone } \\
\text { nt }\end{array}$} & \multirow{2}{*}{ Specifications } & \multicolumn{2}{|c|}{ Dimensions (mm) } \\
\hline & & Bare die & Processed die \\
\hline $\begin{array}{l}\text { MSP430 } \\
\text { MCU }\end{array}$ & $\begin{array}{l}16 \mathrm{MHz}, 16 \mathrm{~KB} \text { Flash, } \\
512 \mathrm{~B} \text { RAM, 10-bit } \\
\text { ADC }\end{array}$ & $\begin{array}{c}2.6 \times 2.5 \times \\
0.280\end{array}$ & $\begin{array}{c}2.6 \times 2.5 \times \\
0.020\end{array}$ \\
\hline Battery & $5 \mu \mathrm{Ah}$ solid state & $\begin{array}{c}1.7 \times 2.25 \times \\
0.200\end{array}$ & $\begin{array}{c}1.7 \times 2.25 \times \\
0.020 \\
\end{array}$ \\
\hline Power & \multicolumn{3}{|c|}{$\begin{array}{l}\text { Low-power System, } 80 \mu \mathrm{A} \text { in active mode, } 3.3 \mathrm{~mA} \text { for } \\
\text { LED }\end{array}$} \\
\hline
\end{tabular}

\section{REFERENCES}

This work has been done at King Abdullah University of Science and Technology (KAUST), Saudi Arabia.

\section{REFERENCES}

[1] S. F. Shaikh, M. T. Ghoneim, G. A. Torres Sevilla, J. M. Nassar, A. M. Hussain, and M. M. Hussain, "Freeform Compliant CMOS Electronic Systems for Internet of Everything Applications," IEEE Trans. Electron Devices, vol. 64, no. 5, 2017, doi: 10.1109/TED.2016.2642340.

[2] J. M. Nassar et al., "Paper Skin Multisensory Platform for Simultaneous Environmental Monitoring," Adv. Mater. Technol., vol. 1, no. 1, p. 1600004, Apr. 2016, doi: 10.1002/admt.201600004.

[3] G. A. Torres Sevilla, M. T. Ghoneim, H. Fahad, J.

P. Rojas, A. M. Hussain, and M. M. Hussain, "Flexible nanoscale high-performance FinFETs," ACS Nano, vol. 8, no. 10, pp. 9850-9856, Oct. 2014, doi: 10.1021/nn5041608.

[4] S. P. Lacour, C. Tsay, and S. Wagner, "An elastically stretchable TFT Circuit," IEEE Electron Device Lett., vol. 25, no. 12, pp. 792-794, Dec. 2004, doi: 10.1109/LED.2004.839227.

[5] Y. Khan et al., "Flexible Hybrid Electronics: Direct Interfacing of Soft and Hard Electronics for Wearable Health Monitoring," Adv. Funct. Mater., vol. 26, no. 47, pp. 8764-8775, 2016, doi: 10.1002/adfm.201603763.

[6] W. Gao et al., "Fully integrated wearable sensor arrays for multiplexed in situ perspiration analysis," Nature, vol. 529, no. 7587, 2016, doi: 10.1038 /nature 16521 .

[7] Z. Huang et al., "Three-dimensional integrated stretchable electronics," Nat. Electron., vol. 1, no. 8, pp. 473-480, 2018, doi: 10.1038/s41928-018-0116$\mathrm{y}$.

[8] Y. Khan, A. E. Ostfeld, C. M. Lochner, A. Pierre, and A. C. Arias, "Monitoring of Vital Signs with Flexible and Wearable Medical Devices," Advanced Materials, vol. 28, no. 22. 2016, doi: 10.1002/adma.201504366.

[9] N. Lu and D.-H. Kim, "Flexible and Stretchable Electronics Paving the Way for Soft Robotics," Soft Robot., vol. 1, no. 1, 2014, doi: 10.1089/soro.2013.0005.

[10] T. Fukushima, Y. Yamada, H. Kikuchi, and M. Koyanagi, "New three-dimensional integration technology using chip-to-wafer bonding to achieve ultimate super-chip integration," Japanese J. Appl. Physics, Part 1 Regul. Pap. Short Notes Rev. Pap., vol. 45, no. 4 B, pp. 3030-3035, 2006, doi: 10.1143/JJAP.45.3030.

[11] J. Viventi et al., "Flexible, foldable, actively multiplexed, high-density electrode array for mapping brain activity in vivo," Nat. Neurosci., vol. 14, no. 12, pp. 1599-1605, 2011, doi: 10.1038/nn.2973.

[12] K. W. Lee, A. Noriki, K. Kiyoyama, T. Fukushima, T. Tanaka, and M. Koyanagi, "Three-dimensional hybrid integration technology of CMOS, MEMS, and photonics circuits for optoelectronic heterogeneous integrated systems," IEEE Trans. Electron Devices, vol. 58, no. 3, pp. 748-757, 2011, 
doi: 10.1109/TED.2010.2099870.

[13] J. Dragas et al., "In Vitro Multi-Functional Microelectrode Array Featuring 59760 Electrodes, 2048 Electrophysiology Channels, Stimulation, Impedance Measurement, and Neurotransmitter Detection Channels," IEEE J. Solid-State Circuits, vol. 52, no. 6, pp. 1576-1590, 2017, doi: 10.1109/JSSC.2017.2686580.

[14] R. R. Bahabry et al., "Corrugation Architecture Enabled Ultraflexible Wafer-Scale High-Efficiency Monocrystalline Silicon Solar Cell," Adv. Energy Mater., vol. 8, no. 12, 2018, doi: 10.1002/aenm.201702221.

[15] S. F. Shaikh, M. T. Ghoneim, R. R. Bahabry, S. M. Khan, and M. M. Hussain, "Modular LegoElectronics," Adv. Mater. Technol., vol. 3, no. 2, p. 1700147, Feb. 2018, doi: 10.1002/admt.201700147.

[16] J. J. S. Norton et al., "Soft, curved electrode systems capable of integration on the auricle as a persistent brain-computer interface.," Proc. Natl. Acad. Sci. U. S. A., vol. 112 , no. 13 , pp. 3920-5, Mar. 2015, doi: 10.1073/pnas. 1424875112 .

[17] Z. Fan et al., "Three-dimensional nanopillar-array photovoltaics on low-cost and flexible substrates," Nat. Mater., vol. 8, no. 8, pp. 648-653, Aug. 2009, doi: $10.1038 /$ nmat 2493 .

[18] C.-H. Lin, W.-T. Hsieh, H.-C. Hsieh, C.-N. Liu, and J.-C. Yeh, "System-level design exploration for 3-D stacked memory architectures," Proc. seventh IEEE/ACM/IFIP Int. Conf. Hardware/software codesign Syst. Synth. - CODES+ISSS '11, p. 389, 2011, doi: 10.1145/2039370.2039435.

[19] N. El-Atab et al., "Heterogeneous Cubic Multidimensional Integrated Circuit for Water and Food Security in Fish Farming Ponds," Small, vol. 16, no. 4, 2020, doi: 10.1002/smll.201905399.

[20] M. Mustafa Hussain and S. F. Shaikh, "HETEROGENOUS INTEGRATION OF PLURAL GRAPHENE SENSORS ON 3D COIN CMOS ELECTRONICS,” PCT/IB2020/051141, 2019.

[21] N. Alcheikh, S. F. Shaikh, and M. M. Hussain, "Inplane deformation mechanics of highly stretchable Archimedean interconnects," AIP Adv., vol. 9, no. 1, p. 015224, Jan. 2019, doi: 10.1063/1.5053967.

[22] A. M. Hussain and M. M. Hussain, "Deterministic Integration of Out-of-Plane Sensor Arrays for

Flexible Electronic Applications," Small, no. 37, pp. 5141-5145, 2016, doi: 10.1002/smll.201600952.

[23] S. F. Shaikh et al., "Noninvasive Featherlight Wearable Compliant 'Marine Skin': Standalone Multisensory System for Deep-Sea Environmental Monitoring," Small, vol. 15, no. 10, p. 1804385 , Mar. 2019, doi: 10.1002/smll.201804385.

[24] J. M. Nassar et al., "Compliant lightweight noninvasive standalone 'Marine Skin' tagging system," npj Flex. Electron., vol. 2, no. 1, p. 13, Dec. 2018, doi: 10.1038/s41528-018-0025-1.

[25] A. M. Hussain, F. A. Ghaffar, S. I. Park, J. A. Rogers, A. Shamim, and M. M. Hussain, "Metal/Polymer Based Stretchable Antenna for Constant Frequency Far-Field Communication in
Wearable Electronics," Adv. Funct. Mater., vol. 25, no. 42, pp. 6565-6575, Nov. 2015, doi: 10.1002/adfm.201503277.

[26] S. F. Shaikh and M. M. Hussain, "Marine IoT: Noninvasive wearable multisensory platform for oceanic environment monitoring," in IEEE 5th World Forum on Internet of Things, WF-IoT 2019 -

Conference Proceedings, Apr. 2019, pp. 309-312, doi: 10.1109/WF-IoT.2019.8767310.

[27] S. F. Shaikh et al., "Noninvasive Featherlight Wearable Compliant 'Marine Skin': Standalone

Multisensory System for Deep-Sea Environmental Monitoring," Small, vol. 15, no. 10, p. 1804385, Mar. 2019, doi: 10.1002/smll.201804385.

[28] S. M. Khan, N. Qaiser, S. F. Shaikh, L. J. Ding, and M. M. Hussain, "Do-It-Yourself integration of a paper sensor in a smart lid for medication

adherence," Flex. Print. Electron., vol. 4, no. 2, p. 025001, Apr. 2019, doi: 10.1088/2058-8585/ab10f5. 


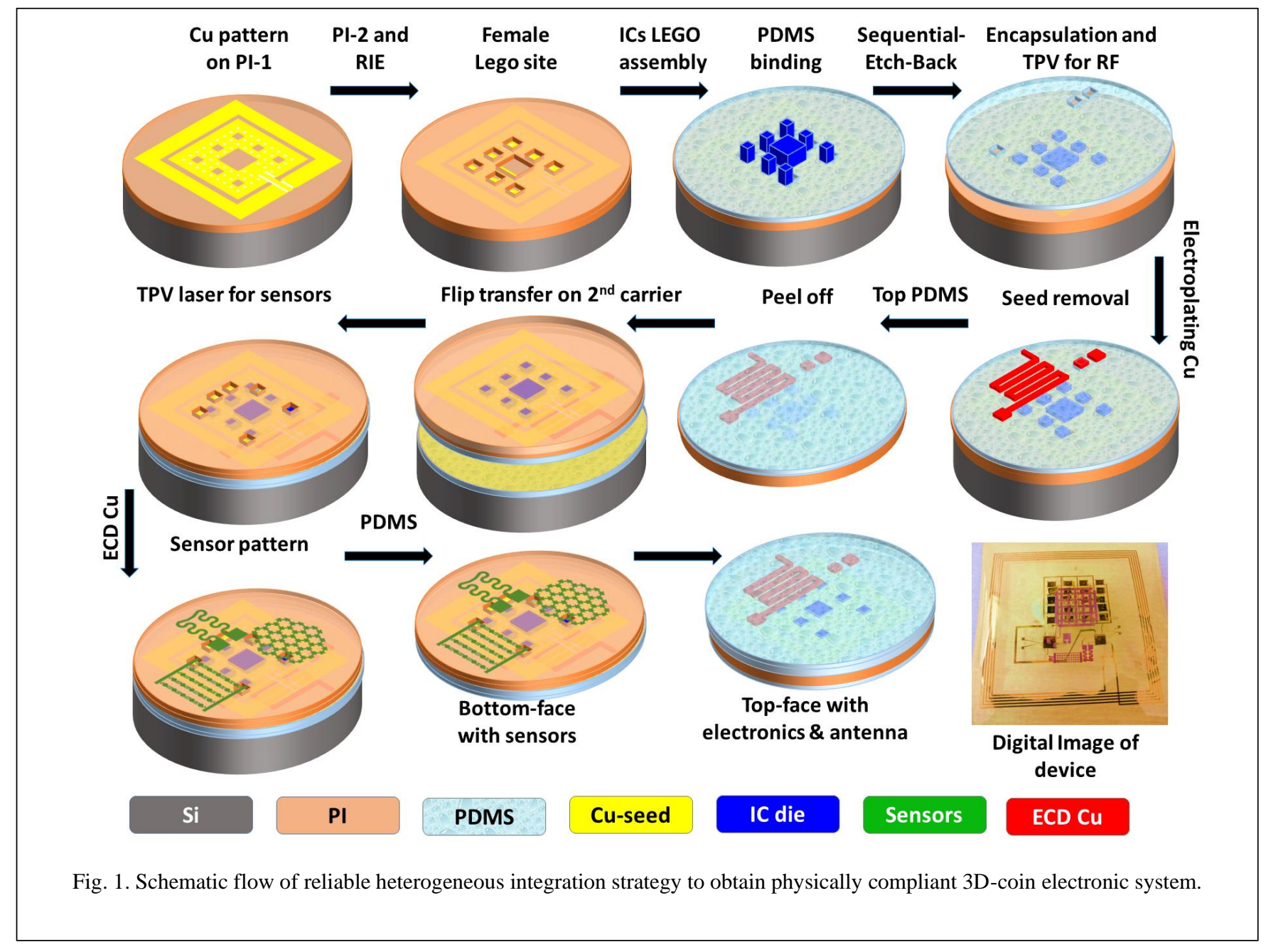



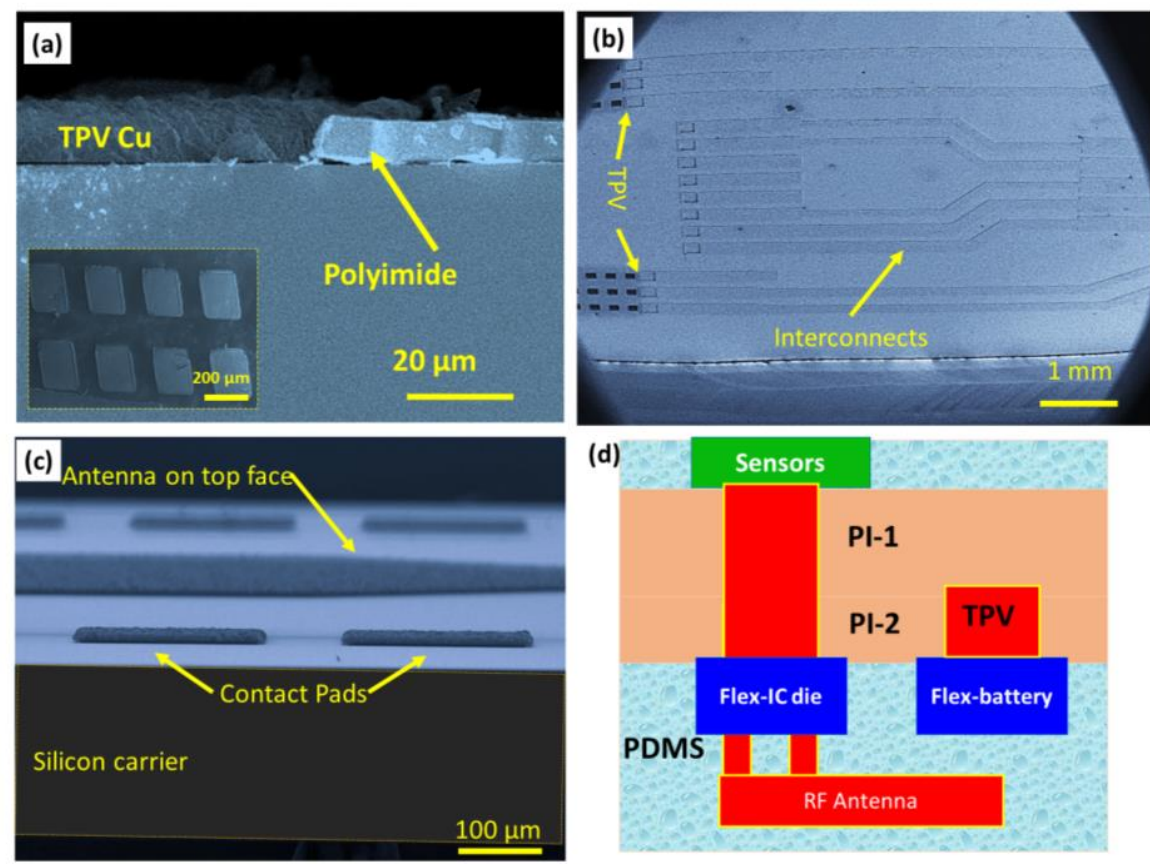

Fig. 2. Scanning electron microscope (SEM) images of (a) TPV filled and edge profile with PI (inset shows multiple TPVs), (b) interconnect profile in the circuit, (c) ECD Cu metallic antenna and connecting pads on top face. (d) The overall 3D cross-sectional view of integration scheme.
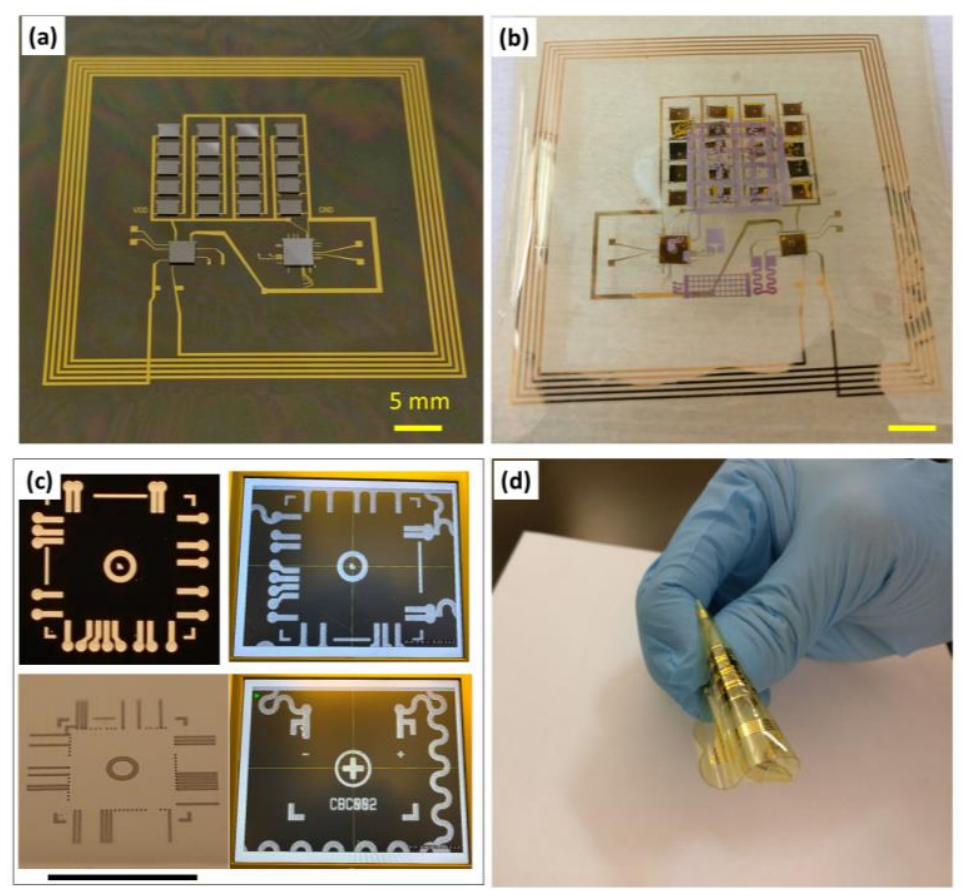

Fig. 3. Integrated system (A) Assembled die array on the platform, (B) Complete 3D coin system after peeling off with sensors and antenna. (C) Microscopic images of LEGO sites and (D) Flexed 3D coin system folded in hand. Scale bar is $5 \mathrm{~mm}$. 
(a)

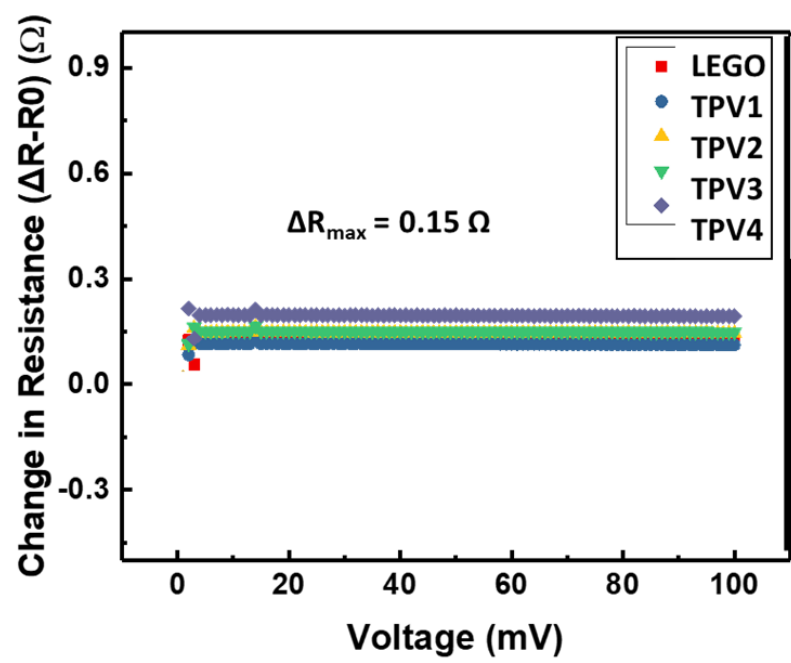

(b)

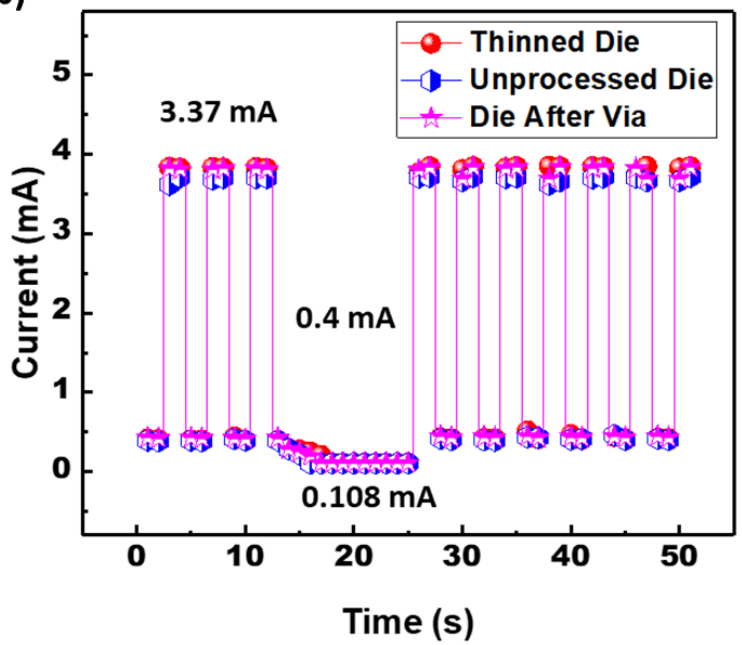

Fig. 4. Electrical characterization of the system. (a) resistance of interconnecting lines and (b) no process induced variation in current consumption.

(a)

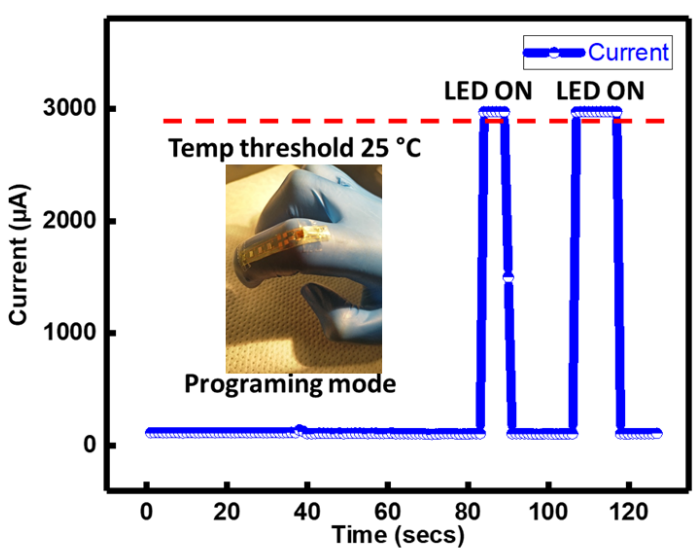

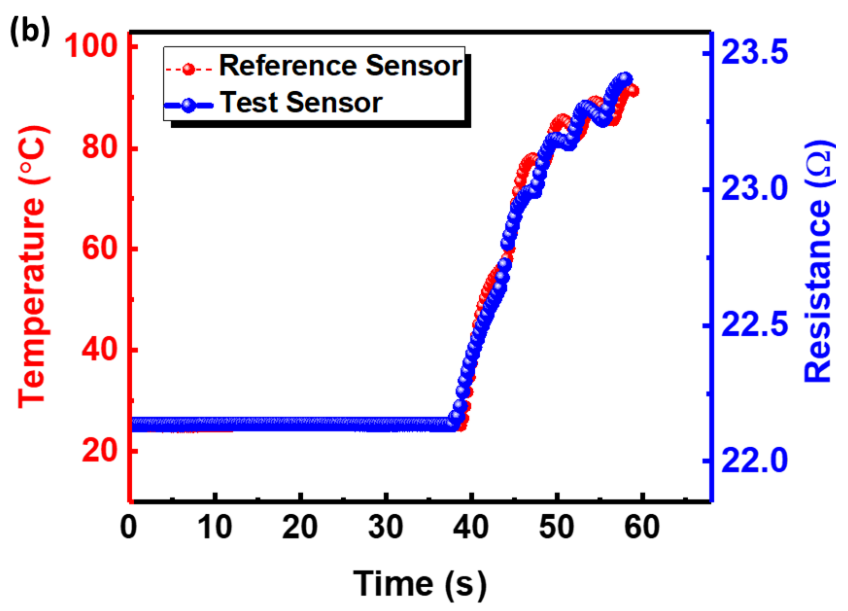

Fig. 5. (a) System demonstration using temperature threshold detection and (b) sensor performance comparing with the reference commercial sensor. 